\title{
Oxytocin receptor (OXTR) polymorphisms and attachment in human infants
}

\author{
Frances S. Chen ${ }^{*}$, Maria E. Barth ${ }^{2}$, Stephen L. Johnson ${ }^{3}$, Ian H. Gotlib ${ }^{4}$ and Susan C. Johnson ${ }^{5 *}$ \\ Department of Psychology, University of Freiburg, Freiburg, Germany \\ 2 Department of Psychology, Tufts University, Boston, MA, USA \\ ${ }_{3}$ Department of Genetics, Washington University Medical School, St. Louis, MO, USA \\ ${ }^{4}$ Department of Psychology, Stanford University, Stanford, CA, USA \\ ${ }^{5}$ Department of Psychology, Ohio State University, Columbus, OH, USA
}

\section{Edited by:}

Daniel Richardson, University College London, UK

\section{Reviewed by:}

Angelica Ronald, Birkbeck College, UK Emma Meaburn, Birkbeck, University of London, UK

\section{*Correspondence:}

Frances S. Chen, Department of Psychology, University of Freiburg, Stefan-Meier-Strasse 8, D-79104 Freiburg, Germany.

e-mail: frances.chen@psychologie. uni-freiburg.de,

Susan S. Johnson, Department of Psychology, The Ohio State University,

Columbus, OH 614-292-8964, USA.

e-mail: johnson.4369@osu.edu

\section{OXYTOCIN RECEPTOR (OXTR) POLYMORPHISMS PREDICT ATTACHMENT IN HUMAN INFANTS}

Humans are social creatures, though some more than others. Even in infancy, humans vary dramatically in their proclivity to seek and accept comfort from caregivers (Bowlby, 1969/1982; Ainsworth et al., 1978). These ordinary variations, readily observable in infancy, are associated with a wide range of individual differences in psychological functioning in adults. Infants who rely on their caregivers with ease and confidence, compared with infants who do not, are more likely to experience stronger cognitive and language development (van IJzendoorn et al., 1995; Sroufe et al., 2005), healthier interpersonal relations (Sroufe et al., 2005), and decreased risk of later mental health problems such as mood disorders, externalizing disorders, and dissociative and attentional disorders (Warren et al., 1997; Carlson, 1998; Greenberg, 1999; Clarke et al., 2002; Sroufe et al., 2005). Although scientists have devoted considerable effort to identifying the foundations of these differences, much remains unknown. In this article we present evidence from molecular genetics demonstrating that at least some of the variance in the quality of infants' attachment behavior is associated with a polymorphism of the oxytocin receptor gene (OXTR).

Attachment is a theory of social stress-regulation, concerned specifically with how infants use caregivers to modulate their own stress. Systematic individual differences in infants' use of caregivers are posited to correspond to infants' expectations and trust of caregivers (Bowlby, 1969/1982; Ainsworth et al., 1978; Johnson et al., 2007). In the standard method for assessing individual differences, known as the Strange Situation (Ainsworth et al., 1978), infants' parent-directed behaviors are observed immediately following a mildly stressful separation and are used to classify infants as either securely or insecurely attached. Securely attached infants tend to readily greet or approach their caregivers; they accept comfort and calm quickly. In contrast, insecurely attached infants either ignore their caregivers or alternately approach and reject them; such infants are slow to calm.

Research on the precursors of infant attachment suggests a role for both environmental and genetic factors. Regarding environmental factors, traditional methods of mother-infant observation have consistently identified parenting behaviors as modest, though robust, sources of variance in the quality of infant attachment. For example, maternal sensitivity and responsiveness has been associated with infant security; similarly, maternal inconsistency, and rejection have been found to be related to infant insecurity (Ainsworth et al., 1978; de Wolff and van Ijzendoorn, 1997). Regarding genetic factors, some behavioral genetic studies on twins suggest that infant attachment also has a heritable component, although other studies have disputed this (see Bakermans-Kranenburg and van IJzendoorn, 2007, for a review of this literature).

Recent advances in molecular genetics permit a different, promising, approach to the examination of genetic influences on attachment by focusing on specific candidate genes. Polymorphisms in genes involved in the production, transport, and metabolism of many neurotransmitters and brain substances have now been related to a variety of mental health outcomes, including psychiatric conditions (Caspi and Moffitt, 2006; Psychiatric GWAS Consortium Coordinating Committee, 2009), as well as to normal variations in stress reactivity (Gotlib et al., 2008) and shyness and behavioral inhibition (Fox et al., 2005). Indeed, several investigators 
have found associations between attachment-relevant behavior and polymorphisms involved in the dopamine and serotonin systems in both adults (Gillath et al., 2008; van IJzendoorn et al., 2008) and children (Bakermans-Kranenburg and van IJzendoorn, 2007; Gervai et al., 2007; Barry et al., 2008), consistent with the role of these neurotransmitters in the regulation of social stress-regulation and approach/withdrawal behaviors.

Another promising target of inquiry is oxytocin and its receptors. Oxytocin is a neuropeptide produced in the hypothalamus and projected via axon transmission into the amygdala and its associated areas where the oxytocin receptor is found (Insel, 1997; Winslow and Insel, 2002). In animals, oxytocin has been found to influence a host of mammalian social behaviors including motherinfant attachment, pair bonding, social recognition, and social communication (Insel, 1997; Winslow and Insel, 2002; Takayanagi et al., 2005; Lim and Young, 2006). Indeed, both oxytocin and oxytocin receptor knockout mice show severe deficits in social behavior (Winslow and Insel, 2002; Takayanagi et al., 2005).

Oxytocin also plays an important role in the social behavior of humans. A single dose of intranasally administered oxytocin has been found to increase healthy adults' attention to the eye region of faces (Guastella et al., 2007), to enhance their ability to infer mental states from eye-related information (Domes et al., 2007), to strengthen their memory for emotional faces (Savaskan et al., 2008), to dampen their negative reactions to aversive faces (Kirsch et al., 2005; Petrovic et al., 2008), and to facilitate their recognition of positive social words (Unkelbach et al., 2008). The effects of oxytocin appear to be specific to social stimuli: compared to controls, adults who received a dose of oxytocin-laden nasal spray accepted greater risk from unseen human partners and trusted them with more of their own money than they did unseen interactive computers (Kosfeld et al., 2005). Finally, Buchheim et al. (2009) reported that the intranasal administration of oxytocin increased the experience of attachment-related security in previously insecure adults.

Oxytocin has also been studied in the context of atypical development. Pollak and his colleagues, for example, found that children with a history of institutionalization and social deprivation showed a smaller rise in urinary oxytocin during an interactive episode with their caregivers than did children without such a history (Wismer Fries et al., 2005). Investigators have also found that intravenous administration of oxytocin improves the social cognition of patients with autism, a neurodevelopmental disorder characterized in part by a lack of social engagement (Hollander et al., 2007).

Polymorphisms exist in the genes for both oxytocin and its receptor. Motivated in part by recent work documenting associations between autism and polymorphisms at several locations within the oxytocin receptor gene (Wu et al., 2005; Jacob et al., 2007; Lerer et al., 2007; Liu et al., 2010), recent studies have examined associations between attachment-related phenomena in adults and single nucleotide polymorphisms (SNPs) within OXTR. The A allele of OXTR rs53576 has been linked to less sensitive parenting behavior (Bakermans-Kranenberg and van Ijzendoorn, 2008), higher stress reactivity (Rodrigues et al., 2009), reduced social support seeking (Kim et al., 2010), and reduced amygdala activation when processing facial emotions (Tost et al., 2010). In one study, the $\mathrm{G}$ allele of rs53576 was associated with insecure attachment in depressed adults (Costa et al., 2009); however, two studies found no association between rs53576 and adults' reports of their own attachment (Gillath et al., 2008; Rodrigues et al., 2009). Another common polymorphism in OXTR, rs2254298, has been linked to amygdala volume in both adults (Inoue et al., 2010) and adolescent girls (Furman et al., 2011), attachment anxiety in adult females and autism-spectrum traits in males (Chen and Johnson, 2011), and depression and anxiety symptoms in adolescent girls (Thompson et al., 2011). However, inconsistent patterns of association between particular alleles (A vs. G) and specific phenotypic outcomes have been reported for rs2254298 (cf. Wu et al., 2005; Jacob et al., 2007).

Considered collectively, the results from animal work, healthy adults, and atypically developing children all point to a probable role of oxytocin in the social development and attachmentrelated behaviors of healthy infants. Although it is not feasible to extract samples of oxytocin from, or administer oxytocin to, healthy infants, the tools of molecular genetics are readily applied to this population. In this context, the goal of the current study was to examine associations between allelic variants at two loci of the oxytocin receptor gene and infant attachment behavior, while taking into consideration possible population differences.

\section{MATERIALS AND METHODS}

Data were obtained from 176 healthy infants ( 98 male) between the ages of 12 and 16 months recruited from a larger study of infant social development in San Francisco Bay Area. Two tested infants for whom DNA extraction failed were not included in the sample. The sample included 77 Caucasians and 99 non-Caucasians. Of the 132 parents who reported family income levels, 46 earned less than the regional median of approximately $\$ 100,000$ annually for a family of 4 , and 86 families earned more. Of the 160 primary caregivers who reported their highest attained educational level, 22 had a high school degree or some college, 80 had a college degree, and 58 had a graduate degree.

Parents reported the child's ethnicity and race using the categories developed by the National Institute of Health for reporting purposes. These include two ethnic categories (Hispanic or Latino, and Not Hispanic or Latino) and five racial categories (American Indian or Alaska Native, Asian, Black or African American, Native Hawaiian or Other Pacific Islander, and White). For our purposes, infants reported to be Non-Hispanic and White were classified as "Caucasian," and all others as "non-Caucasian." Because the allele frequencies in Caucasian populations that have been studied to date differ significantly from those documented in all other populations studied to date ${ }^{1}$, analyses combining data from Caucasian and non-Caucasian infants introduce the potential confound of population stratification due to genetic drift. Thus, we also included ethnicity (Caucasian and non-Caucasian) in our analyses. Although "non-Caucasian" does not designate a genetically homogenous population, very similar allele frequencies at rs2254298 have been documented in the three non-Caucasian populations that have been studied to date (see text footnote 1).

${ }^{1}$ www.hapmap.org 
All parents gave their written informed consent. The study was approved by the Institutional Review Board of Stanford University. All statistical analyses were conducted using SPSS18 (SPSS, Inc.).

\section{ATTACHMENT STATUS}

The quality of each infant's attachment to his/her primary caregiver was assessed using the Strange Situation, a standardized procedure and set of coding criteria developed by Ainsworth et al. (1978). This procedure guides the infant through a series of 3 min episodes in which he or she is first brought, with his or her caregiver, to an unfamiliar room filled with toys, then introduced to a stranger, left alone with the stranger, and finally left entirely alone. After each separation the infant is reunited with either the stranger or his or her caregiver. Infants' approach, physical contact, resistant, and avoidant behaviors toward the caregiver in reunion episodes were used to classify infants as either secure or insecure (Ainsworth et al., 1978). The primary coder was trained and certified at the Minnesota Institute for Child Development Attachment Workshop. A secondary coder double-coded $85(48 \%)$ of the tapes, $\kappa=0.70$.

\section{GENOTYPING}

Two SNPs were selected for genotyping on the basis of their known association with autism as well as with attachment-related outcomes in healthy adults: OXTR rs2254298 and OXTR rs53576. Both are located in the third intron of OXTR and are likely non-coding markers of unidentified functional variations in the oxytocin receptor gene.

DNA was collected from saliva samples using the Oragene Kit (DNA Genotek, Ottawa, ON, Canada). Amplification of rs2254298 was performed with the primers 5'-TGAAAGCAGAGGTTGTGTGGACAGG-3' and the antisense primer 5'-AACGCCCACCCCAGTTTCTTC-3' under standard conditions. Amplification of rs53576 was performed with the primers GCCCACCATGCTCTCCACATC and GCTGGACTCAGGAGGAATAGGGAC. Call rates were $\sim 98 \%$ for each SNP. Three samples that failed on the first attempt were successfully re-run. Genotyping was carried out by KBiosciences using their internal KASP chemistry, a form of fluorescence-based competitive allele-specific PCR using FRET quencher cassette oligonucleotides. Further details of the assay design are available directly from KBiosciences ${ }^{2}$.

\section{RESULTS}

\section{ATTACHMENT STATUS}

Ninety-eight of the infants (56\%) were classified as secure and $78(44 \%)$ as insecure. Preliminary analyses yielded no effects of family income or caregiver's education on the rate of security; consequently, these variables were removed from further analysis.

\section{GENOTYPING}

Allele and genotype frequencies of both SNPs are summarized in Table 1. Both SNPs were in Hardy-Weinberg equilibrium for the overall sample as well as the Caucasian and non-Caucasian subsamples (all ps >0.47). The allele frequencies in our Caucasian and non-Caucasian samples are consistent with those reported

${ }^{2} \mathrm{http} / / /$ www.kbioscience.co.uk in the human genome database (see text footnote 1), Jacob et al. (2007), and Wu et al. (2005). For both SNPs, the A allele was more common in the non-Caucasian samples than in the Caucasian samples. To test for association between the two SNPs, we used Haploview $4.2^{3}$ to calculate $r^{2}$, a measure of linkage disequilibrium. In our overall sample as well as within the two ethnic subsamples, negligible linkage disequilibrium was observed (all $\left.r^{2}<0.01\right)$.

For rs53576, preliminary analyses using an additive genetic model revealed no significant effects. Therefore, a dominant genetic model (grouping infants based on the presence or absence of at least one A allele) was also tested; this model has also been used in previous studies showing associations between rs53576 and social behavior in humans (Bakermans-Kranenberg and van Ijzendoorn, 2008; Rodrigues et al., 2009). For rs2254298, testing the additive genetic model would have been uninformative given the rarity of the AA carriers - only five AA carriers (3\%) were present in the sample. Therefore, we used a dominant genetic model, combining the AA and AG carriers creating into a single group defined by having at least one A allele. Eliminating the AA carriers entirely from the analyses to leaving a comparison of only AG vs. GG carriers (the strategy taken in Thompson et al., 2011) does not significantly change any of the reported results.

\section{OXTR rs53576}

A dominant genetic model was tested, with infants grouped according to the presence $(n=103)$ or absence $(n=73)$ of at least one A allele. Consistent with the previous finding in adults of Gillath et al. (2008), we found no association between variants of rs53576 and security of attachment, even when accounting for ethnicity and sex, as tested by a logistic regression with the terms entered together: rs53576 (presence vs. absence of the A allele), ethnicity (Caucasian vs. non-Caucasian), sex, and their interactions, all $p s>0.45$.

\section{OXTR rs2254298}

Grouping the infants according to the presence of at least one A allele resulted in 47 infants with and 129 without. As with other findings in the literature (Wu et al., 2005; Jacob et al., 2007), the association between the genotype at rs2254298 (presence vs.

${ }^{3}$ http://www.broad.mit.edu/mpg/haploview

Table 1 | Allele and genotype frequencies of OXTR SNPs.

\begin{tabular}{|c|c|c|c|c|c|}
\hline & \multicolumn{2}{|c|}{ Allele distribution } & \multicolumn{3}{|c|}{ Genotype distribution } \\
\hline & $\mathbf{G}$ & A & GG & AG & AA \\
\hline \multicolumn{6}{|l|}{ RS53576 } \\
\hline Overall sample & $224(64 \%)$ & $128(36 \%)$ & $73(41 \%)$ & $78(44 \%)$ & $25(14 \%)$ \\
\hline Caucasians & $114(74 \%)$ & $40(26 \%)$ & $41(53 \%)$ & $32(42 \%)$ & $4(5 \%)$ \\
\hline Non-Caucasians & $110(56 \%)$ & $88(44 \%)$ & $32(32 \%)$ & $46(46 \%)$ & $21(21 \%)$ \\
\hline \multicolumn{6}{|l|}{ RS2254298 } \\
\hline Overall sample & $300(85 \%)$ & $52(15 \%)$ & $129(73 \%)$ & $42(24 \%)$ & $5(3 \%)$ \\
\hline Caucasians & $141(92 \%)$ & $13(8 \%)$ & $65(84 \%)$ & $11(14 \%)$ & $1(1 \%)$ \\
\hline Non-Caucasians & $159(80 \%)$ & $39(20 \%)$ & $64(65 \%)$ & $31(31 \%)$ & $4(4 \%)$ \\
\hline
\end{tabular}


absence of the A allele) and the behavioral outcome (secure vs. insecure) appeared to differ as a function of ethnicity (Caucasian vs. non-Caucasian), with the opposite directions of association in the two groups (Figure 1). This interaction was confirmed with a logistic regression in which genotype, ethnicity, sex, and their possible interactions were entered together, which remains significant $(p<0.05)$ after accounting for multiple testing of two SNPs (rs53576 and rs2254298) using a Bonferroni correction.

To examine the association between rs2254298 and security of attachment further, we conducted further separate regression analyses on each population. Because no effects for sex were seen, it was removed from these further analyses. We also performed post hoc power estimations for the logistic regression analyses of both the Caucasian and non-Caucasian samples using the power calculator for logistic regressions at http://www.dartmouth.edu/ eugened/ power-samplesize.php.

\section{OXTR RS2254298 ANALYSIS BY ETHNICITY}

\section{Caucasians}

Six of the 12 Caucasian infants (50\%) with the A allele and 39 of the 65 Caucasian infants (60\%) without the A allele were securely attached to their caregivers. A logistic regression detected no association between the presence of an A allele at rs2254298 and the attachment status of the Caucasian infants, $p>0.50$ (see Table 2). Given our sample size and observed effect size, the power to detect a significant (at the 5\% level) main effect of rs2254298 (dominant model) on attachment status in Caucasians was less than $1 \%$.

\section{NON-CAUCASIANS}

In contrast to the Caucasians, 26 of the 35 non-Caucasians infants (74\%) with the A allele were securely attached compared to only 27 of the 64 infants ( $42 \%$ ) without it. Thus, the non-Caucasian infants were almost four times more likely to develop a secure attachment

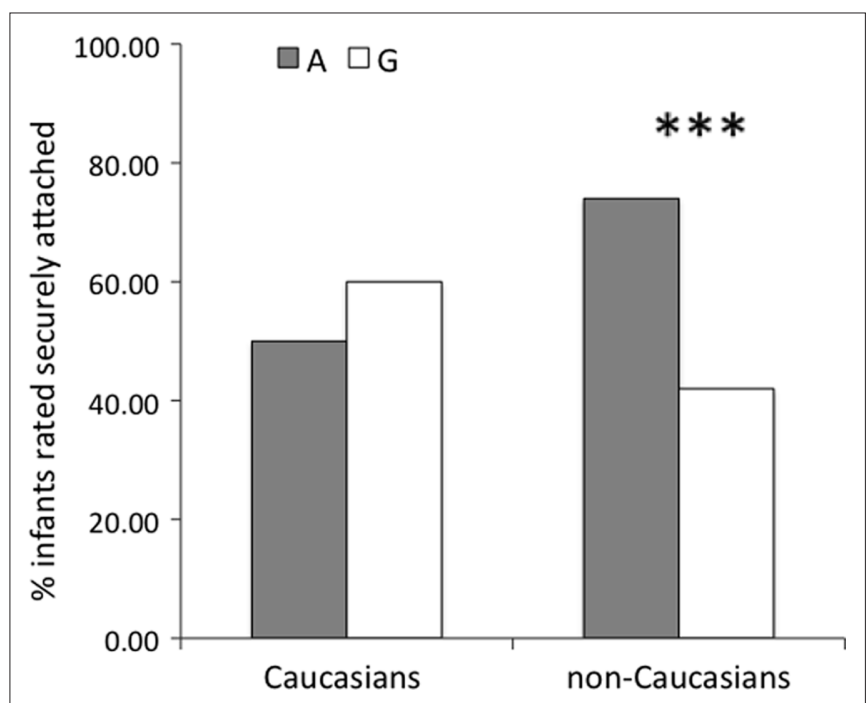

FIGURE 1 | Percentage of infants classified as secure as a function of rs2254298 genotype and ethnicity. Group $A$ includes infants with the AA or AG genotype. Group $G$ includes infants with the GG genotype. The effect of genotype on attachment security was significant in the non-Caucasian group $(p<0.005)$. if they had the A allele at rs2254298 than if they did not, $p=0.003$ by a logistic regression (see Table 2 ). Given our sample size and observed effect size, the power to detect a significant (at the 5\% level) main effect of rs2254298 (dominant model) on attachment status in non-Caucasians was $65 \%$.

\section{DISCUSSION}

These results are the first to document a specific association between a polymorphism in the oxytocin receptor gene and individual differences in the security of attachment in a population of human infants. The association suggests that there are at least two functional variants of the oxytocin receptor gene. The variant marked by the A allele at rs2254298 was significantly more likely to be associated with secure attachment than was the variant marked by the $\mathrm{G}$ allele in non-Caucasian infants, but not in Caucasian infants. These non-Caucasian infants were more willing and able to accept comfort from their caregivers if they had at least one A allele at rs2254298 than if they did not. As a group, Caucasian infants showed no associations between the rs2254298 SNP and security of attachment. No associations were found with the rs53576 SNP in any ethnic group.

It is not clear why there was not a significant association between OXTR and attachment in the Caucasian infants. It is noteworthy, however, that previous studies have documented ethnic differences in OXTR associations with phenotype. In the Chinese Han population studied by Wu et al. (2005), the A allele of rs2254298 was associated with autism. In contrast, in the Caucasian sample studied by Jacob et al. (2007), the G allele was associated with autism. One explanation for this discrepancy involves patterns of linkage disequilibrium between the functional loci and the associated markers used in these studies. In evolutionary time, patterns of linkage disequilibrium naturally change through the recurring process of recombination. When two populations diverge, the patterns of linkage disequilibrium within each may also diverge. It is possible, therefore, that a functional SNP of OXTR is linked to the rs2254298 A allele in the Chinese Han population, but the $G$ allele in the Caucasian population. These patterns of linkage disequilibrium would be consistent with the observation that Caucasian infants are not less likely to be securely attached than non-Caucasian infants despite the relatively low frequency of the A allele in Caucasian populations (see text footnote 1).

Even without differences in patterns of linkage disequilibrium, possible interactions with other genetic or cultural factors may affect our ability to detect oxytocin receptor function in some populations more than in others. For instance, an important role of oxytocin receptors is to regulate serotonin (Yoshida et al., 2009) and dopamine function (Liu and Wang, 2003). Serotonin and dopamine function, however, are influenced by multiple factors, including

Table 2 | Logistic regressions predicting security of attachment from presence or absence of the A allele at OXTR rs2254298 for Caucasian and non-Caucasian populations.

\begin{tabular}{lllll}
\hline Population & $\boldsymbol{B}$ & Wald $\chi^{2}$ & $\boldsymbol{p}$ & Odds ratio [95\% Cl] \\
\hline Caucasians & -0.40 & 0.41 & 0.52 & $0.67[0.19,2.29]$ \\
Non-Caucasians & 1.38 & 8.86 & 0.003 & $3.95[1.60,9.80]$
\end{tabular}


other genetic polymorphisms that themselves vary across ethnic populations (e.g., Chang et al., 1996; Kunugi et al., 1997). These unmeasured differences may mask oxytocin function and inhibit our ability to observe variations in OXTR function to a greater extent in some populations than in others.

We may not be able to resolve these issues until we gain a more complete understanding of the structure of the oxytocin receptor gene. In the meantime, obtaining information about additional OXTR SNPs in association with behavioral data should allow us to identify haplotypes (chromosomes characterized by specific permutations of multiple SNPs) within these initial groupings that may have even stronger associations with particular attachment phenotypes. In any case, the differences between Caucasian and non-Caucasian infants documented in this study serve as a reminder of the difficulties involved in generalizing genetic contributions across populations.

As with all genetic association studies, our finding should be replicated in an independent sample in order to establish the robustness of the effect observed in our sample as well as to clarify its effect size. Further studies of the role of OXTR in infant behavior would be of particular relevance, as all previous studies have tested adult samples. Given the broad confidence interval of our reported effect in non-Caucasians, the inclusion of larger, more homogeneous samples in future research would be advisable. In Caucasians, it remains to be seen whether future studies using a much larger sample will establish a significant, reversed pattern of association between the alleles studied and attachment status (comparable to the opposite patterns of association in different ethnic groups between specific alleles at rs2254298 and autism risk observed in previous research).

Attachment behaviors in infants represent a relatively gross behavioral manifestation of infants' underlying cognitive and emotional processes. Further examination of finer-grained aspects of social information processing and behavior is needed. Not only will this advance our understanding of attachment processes, but it may also facilitate the identification of additional possible allelic

\section{REFERENCES}

Ainsworth, M. D.S., Blehar, M.C., Waters, E., and Wall, S. (1978). Patterns of Attachment: A Psychological Study of the Strange Situation. Hillsdale, NJ: Erlbaum.

Bakermans-Kranenberg, M. J., and van Ijzendoorn, M. H. (2008). Oxytocin receptor (OXTR) and serotonin transporter (5-HTT) genes associated with observed parenting. Soc. Cogn. Affect. Neurosci. 3, 128-134.

Bakermans-Kranenburg, M. J., and van IJzendoorn, M. H. (2007). Research review: genetic vulnerability and differential susceptibility in child development: the case of attachment. J. Child Psychol. Psychiatry 48, 1160-1173.

Barry, R. A., Kochanska, G., and Philibert, R. A. (2008). G x E interaction in the organization of attachment: mothers' responsiveness as a

variants of OXTR. Closer examination of these building blocks of social information processing may also help clarify possible associations among OXTR alleles, attachment, and autism in individuals of different ethnicities.

One limitation of the current study is that we did not measure either parental behavior or genotype. Nonetheless, based on other examples in the developmental literature (Fox et al., 2005; Caspi and Moffitt, 2006; Bakermans-Kranenburg and van IJzendoorn, 2007; Barry et al., 2008), and on work examining parenting and gene expression in rodents (Meaney, 2001), we expect that future studies will find that OXTR interacts with caregiving to predict infant attachment. In addition, it is possible that the current results are an indirect reflection of the parents' genotype, particularly given Bakermans-Kranenberg and van Ijzendoorn's (2008) finding that OXTR is related to parenting style. It should be noted however, that whereas Bakersman-Kranenberg and van Ijzendoorn found an association between parenting and rs53576, the association reported here is with rs2254298. We did not find an association between attachment status and rs53576.

The identification of different functional variants of the oxytocin receptor gene sets the stage for beginning to examine causal differences among alleles. These differences may lie in the functional activity of the gene product itself, in the levels of its expression in different brain areas or at different times, or in the sensitivity of its expression to environmental influences. A clearer understanding of this biology promises to help us elucidate mechanisms of social stress-regulation that lead to individual differences in both adaptive and maladaptive psychological functioning.

\section{ACKNOWLEDGMENTS}

The authors thank Karen Parker and Anna Penn for their discussions on the role of oxytocin in infancy and Joachim Hallmayer for help with an earlier version of this work. Funding: This research was supported by a Hass Foundation seed grant to Susan C. Johnson and by Grant MH074849 from the National Institute of Mental Health to Ian H. Gotlib.

The world-wide distribution of allele frequencies at the human dopamine D4 receptor locus. Hum. Genet. 98, 91-101.

Chen, F. S., and Johnson, S. C. (2011). An oxytocin receptor gene variant predicts attachment anxiety in females and autism-spectrum traits in males. Soc. Psychol. Personal. Sci. doi: 10.1177/1948550611410325

Clarke, L., Ungerer, J. A., Chahoud, K., Johnson, S., and Stiefel, I. (2002). Attention deficit hyperactivity disorder is associated with attachment insecurity. Clin. Child Psychol. Psychiatry 7, 179-198.

Costa, B., Pini, S., Gabelloni, P., Abelli, M., Lari, L., Cardini, A., Muti, M., Gesi, C., Landi, S., Galderisi, S., Mucci, A., Lucacchini, A., Cassano, G. B., and Martini, C. (2009). Oxytocin receptor polymorphisms and adult attachment style in patients with depression. Psychoneuroendocrinology 34, 1506-1514.

de Wolff, M. S., and van Ijzendoorn, M. H. (1997). Sensitivity and attachment: a meta-analysis on parental antecedents of infant attachment. Child Dev. 68, 571-591.

Domes, G., Heinrichs, M., Michel, A., Berger, C., and Herpertz, S. C. (2007). Oxytocin improves "mind-reading" in humans. Biol. Psychiatry 61, 731-733. Fox, N. A., Nichols, K. E., Henderson, H. A., Rubin, K., Schmidt, L., Hamer, D., Ernst, M., and Pine, D. S. (2005). Evidence for a gene-environment interaction in predicting behavioral inhibition in middle childhood. Psychol. Sci. 16, 921-926.

Furman, D. J., Chen, M. C., and Gotlib, I. H. (2011). Variant in oxytocin receptor gene is associated with amygdala volume. Psychoneuroendocrinology 36 , 891-897. 
Gervai, J., Novak, A., Lakatos, K., Toth, I., Danis, I., Ronai, Z., Nemoda, Z., Sasvari-Szekely, M., Bureau, J. F., Bronfman, E., and Lyons-Ruth, K. (2007). Infant genotype may moderate sensitivity to maternal affective communications: attachment disorganization, quality of care, and the DRD4 polymorphism. Soc. Neurosci. 2,307-319.

Gillath, O., Shaver, P. R., Baek, J.-M., and Chun, D. S. (2008). Genetic correlates of adult attachment style. Pers. Soc. Psychol. Bull. 34, 1396-1405.

Gotlib, I. H., Joormann, J., Minor, K. L., and Hallmayer, J. (2008). HPA-Axis reactivity may underlie the associations among the 5-HTTLPR polymorphism, stress, and risk for depression. Biol. Psychiatry 63, 847-851.

Greenberg, T. (1999). "Attachment and psychopathology in childhood," in Handbook of Attachment, eds J.Cassidy and P. R. Shaver (New York: Guilford), 269-296.

Guastella, A. J., Mitchell, P. B., and Dadds, M. R. (2007). Oxytocin increases gaze to the eye region of human faces. Biol. Psychiatry 63, 3-5.

Hollander, E., Bartz, J., Chaplin, W., Phillips, A., Sumner, J., Soorya, L., Anagnostou, E., and Wasserman, S. (2007). Oxytocin increases retention of social cognition in autism. Biol. Psychiatry 61, 498-503.

Inoue, $\mathrm{H}$., Yamasue, H., Tochigi, M., Abe, O., Liu, X., Kawamura, Y., Takei, K., Suga, M., Yamada, H., Rogers, M. A., Aoki, S., Sasaki, T., and Kasai, K. (2010). Association between the oxytocin receptor gene and amygdalar volume in healthy adults. Biol. Psychiatry 68, 1066-1072.

Insel, T. R. (1997). A neurobiological basis of social attachment. Am. J. Psychiatry 154, 726-735.

Jacob, S., Brune, C. W., Carter, C. S., Leventhal, B. L., Lord, C., and Cook, E. H. (2007). Association of the oxytocin receptor gene (OXTR) in Caucasian children and adolescents with autism. Neurosci. Lett. 417, 6-9.

Johnson, S. C., Dweck, C. S., and Chen, F. S. (2007). Evidence for infants' internal working model of attachment. Psychol. Sci. 18, 501-502.

Kim, H. S, Sherman, D. K., Sasaki, J. Y., Xu, J., Chu, T. Q., Ryu, C., Suh, E. M., Graham, K., and Taylor, S. E. (2010). Culture, distress and oxytocin receptor polymorphism (OXTR) interact to influence emotional support seeking. Proc. Natl. Acad. Sci. U.S.A. 107, 15717-15721.

Kirsch, P., Esslinger, C., Chen, Q., Mier, D., Lis, S., Siddhanti, S., Gruppe, H., Mattay, V.S., Gallhofer, B., and MeyerLindenberg, A. (2005). Oxytocin modulates neural circuitry for social cognition and fear in humans. $J$. Neurosci. 25, 11489-11493.

Kosfeld, M., Heinrichs, M., Zak, P. J., Fischbacher, U., and Fehr, E. (2005). Oxytocin increases trust in humans. Nature 435, 673-676.

Kunugi, H. K., Hattori, M., Kato, T., Tatsumi, M., Sakai, T., Sasaki, T., Hirose, T., and Nanko, S. (1997). Serotonin transporter gene polymorphisms: ethnic difference and possible association with bipolar affective disorder. Mol. Psychiatry 2, 457-462.

Lerer, E., Levi, S., Salomon, S., Darvasi, A., Yirmiya, N., and Ebstein, R. P. (2007). Association between the oxytocin receptor (OXTR) gene and autism: relationship to Vineland Adaptive Behavior Scales and cognition. Mol. Psychiatry 13, 980-988.

Lim, M. M., and Young, L. J. (2006). Neuropeptidergic regulation of affiliative behavior and social bonding in animals. Horm. Behav. 50, 506-517.

Liu, X., Kawamura, Y., Shimada, T., Otawa, T., Koishi, S., Sugiyama, T., Nishida, H., Hashimoto, O., Nakagami, R., Tochigi, M., Umekage, T., Kano, Y., Miyagawa, T., Kato, N., Tokunaga, K., and Sasaki, T. (2010). Association of the oxytocin receptor (OXTR) gene polymorphisms with autism spectrum disorder (ASD) in the Japanese population. J. Hum. Genet. 55, 137-141.

Liu, Y., and Wang, Z. X. (2003). Nucleus accumbens oxytocin and dopamine interact to regulate pair bond formation in female prairie voles. Neuroscience 121, 537-544.

Meaney, M. J. (2001). Maternal care, gene expression, and the transmission of individual differences in stress reactivity across generations. Annu. Rev. Neurosci. 24, 1161-1192.

Petrovic, P., Kalisch, R., Singer, T., and Dolan, R. J. (2008). Oxytocin attenuates affective evaluations of conditioned faces and amygdala activity. $J$. Neurosci. 28, 6607-6615.

Psychiatric GWAS Consortium Coordinating Committee. (2009). Genomewide association studies: history, rationale, and prospects for psychiatric disorders. Am. J. Psychiatry $166,540-556$.

Rodrigues, S. M., Saslow, L. R., Garcia, N., John, O. P., and Keltner, D. (2009). Oxytocin receptor genetic variation relates to empathy and stress reactivity in humans. Proc. Natl. Acad. Sci. U.S.A. 106, 21437-21441.

Savaskan, E., Ehrhardt, R., Schulz, A., Walter, M., and Schachinger, H. (2008). Post-learning intranasal oxytocin modulates human memory for facial identity. Psychoneuroendocrinology 33 , 368-374.

Sroufe, L. A., Egeland, B., Carlson, E., and Collins, W. A. (2005). "Placing early attachment experiences in developmental context: the Minnesota longitudinal study," in Attachment from Infancy to Adulthood, eds K. E. Grossmann, K. Grossmann, and E. Waters (New York: Guilford), 48-70.

Takayanagi, Y., Yoshida, M., Bielsky, I. F. Ross, H. E., Kawamata, M., Onaka, T., Yanagisawa, T., Kimura, T., Matzuk, M. M., Young, L. J., and Nishimori, K. (2005). Pervasive social deficits, but normal parturition, in oxytocin receptor-deficient mice. Proc. Natl. Acad. Sci. U.S.A. 102, 16096-16101.

Thompson, R. J., Parker, K. J., Hallmayer, J. F., Waugh, C. E., and Gotlib, I. H. (2011). Oxytocin receptor gene polymorphism (rs2254298) interacts with familial risk for psychopathology to predict symptoms of depression and anxiety in adolescent girls. Psychoneuroendocrinology 36, 144-147.

Tost, H., Kolachana, B., Hakimi, S. Lemaitre, H., Verchinski, B. A., Mattay, V. S., and Weinberger, D. R. (2010). A common allele in the oxytocin receptor gene (OXTR) impacts prosocial temperament and human hypothalamic-limbic structure and function. Proc. Natl. Acad. Sci. U.S.A. 107, 13936-13941.

Unkelbach, C., Guastella, A. J., and Forgas, J. P. (2008). Oxytocin selectively faciliatates recognition of positive sex and relationship words. Psychol. Sci. 19 1092-1094.

van IJzendoorn, M. H., BakermansKranenburg, M. J., and Mesman, J. (2008). Dopamine system genes associated with parenting in the context of daily hassles. Genes Brain Behav. 7, 403-410.

van IJzendoorn, M. H., Dijkstra, J., and Bus, A. G. (1995). Attachment, intelligence, and language. Soc. Dev. 4, 115-128.

Warren, S. L., Huston, L., Egeland, B., and Sroufe, L. A. (1997). Child and adolescent anxiety disorders and early attachment.J.Am. Acad. Child Adolesc. Psychiatry 36, 637-644.

Winslow, J. T., and Insel, T. R. (2002). The social deficits of the oxytocin knockout mouse. Neuropeptides 36, 221-229.

Wismer Fries, A. B., Zigler, T. E., Kurian, J. R., Jacoris, S., and Pollak, S. D. (2005). Early experience in humans is associated with changes in neuro-peptides critical for regulating social behaviour. Proc. Natl. Acad. Sci. U.S.A. 102, $17237-17240$.

Wu, S., Jia, M., Ruan, Y., Liu, J., Guo, Y., Shuang, M., Gong, X., Zhang, Y., Yang, X., and Xhang, D. (2005). Positive association of the oxytocin receptor gene (OXTR) with autism in the Chinese Han population. Biol. Psychiatry 58, 74-77.

Yoshida, M., Takayanagi, Y., Inoue, I., Kimura, T., Young, L. J., Onaka, T., and Nishimori, K. (2009). Evidence that oxytocin exerts anxiolytic effects via oxytocin receptor expressed in serotonergic neurons in mice. J. Neurosci. 29, 2259-2271.

Conflict of Interest Statement: The authors declare that the research was conducted in the absence of any commercial or financial relationships that could be construed as a potential conflict of interest.

Received: 07 July 2010; accepted: 08 August 2011; published online: 25 August 2011.

Citation: Chen FS, Barth ME, Johnson SL, Gotlib IH and Johnson SC (2011) Oxytocin receptor (OXTR) polymorphisms and attachment in human infants. Front. Psychology 2:200. doi: 10.3389/ fpsyg.2011.00200

This article was submitted to Frontiers in Developmental Psychology, a specialty of Frontiers in Psychology.

Copyright $\odot 2011$ Chen, Barth, Johnson, Gotlib and Johnson. This is an open-access article subject to a non-exclusive license between the authors and Frontiers Media $S A$, which permits use, distribution and reproduction in other forums, provided the original authors and source are credited and other Frontiers conditions are complied with. 